CERN-TH/2003-250

\title{
Solution of the Dirac equation in lattice QCD using a domain decomposition method
}

\author{
Martin Lüscher \\ CERN, Theory Division \\ CH-1211 Geneva 23, Switzerland
}

\begin{abstract}
Efficient algorithms for the solution of partial differential equations on parallel computers are often based on domain decomposition methods. Schwarz preconditioners combined with standard Krylov space solvers are widely used in this context, and such a combination is shown here to perform very well in the case of the Wilson-Dirac equation in lattice QCD. In particular, with respect to even-odd preconditioned solvers, the communication overhead is significantly reduced, which allows the computational work to be distributed over a large number of processors with only small parallelization losses.
\end{abstract}

\section{Introduction}

At present, numerical simulations of lattice QCD including sea-quark effects are still limited to relatively small lattices and large quark masses. The rapid increase in the computer power that is available for these calculations will certainly help to improve the situation, but it may also be necessary to develop better algorithms to be able to reach the chiral regime, where the effects associated with the spontaneous breaking of chiral symmetry become important.

In this connection the applicability of domain decomposition methods $[1,2]$ seems worth studying, given the fact that the standard formulations of lattice QCD involve nearest-neighbour interactions only. A first step in this direction was made in ref. [3], where a simulation algorithm for two-flavour lattice QCD was proposed, which can 
be regarded as an implementation of the classic Schwarz alternating procedure [4] at the quantum level.

The classic Schwarz procedure may also be used directly for the solution of the lattice Dirac equation, and the aim in the present paper is to show that this leads to a competitive solver, particularly so if the computational work is to be distributed over a large number of nodes of a parallel computer. While this result is immediately relevant to the QCD simulation algorithms that are currently in use, the study of the Schwarz procedure in this context also serves as a preparation for the implementation of the proposed Schwarz simulation algorithm [3].

For simplicity only the standard Wilson formulation of lattice QCD [5] will be considered, but $\mathrm{O}(a)$ improvement [6,7] can easily be included and the basic strategies are in any case expected to be more generally applicable. The Schwarz procedure for the solution of the lattice Dirac equation looks fairly natural in this framework and is explained in all detail. By itself this method is not very efficient, but it can be combined, in the form of a preconditioner for the Wilson-Dirac operator, with the GCR Krylov space accelerator. The excellent performance of the resulting algorithm is then demonstrated by a series of test runs on a recent PC-cluster with 64 processors.

\section{Schwarz alternating procedure}

\subsection{Lattice Dirac operator}

As usual the fields in lattice QCD are assumed to live on the sites $x$ of a hypercubic four-dimensional lattice of finite extent. Periodic boundary conditions are imposed in all directions and the lattice spacing is set to unity for notational convenience.

On the lattice the $\mathrm{SU}(3)$ gauge field is represented by group-valued link variables $U(x, \mu), \mu=0, \ldots, 3$, while the quark fields $\psi(x)$ carry Dirac and colour indices as in the continuum theory. The gauge-covariant forward and backward difference operators are then given by

$$
\begin{aligned}
& \nabla_{\mu} \psi(x)=U(x, \mu) \psi(x+\hat{\mu})-\psi(x), \\
& \nabla_{\mu}^{*} \psi(x)=\psi(x)-U(x-\hat{\mu}, \mu)^{-1} \psi(x-\hat{\mu}),
\end{aligned}
$$

where $\hat{\mu}$ denotes the unit vector in direction $\mu$, and using these the Wilson-Dirac 


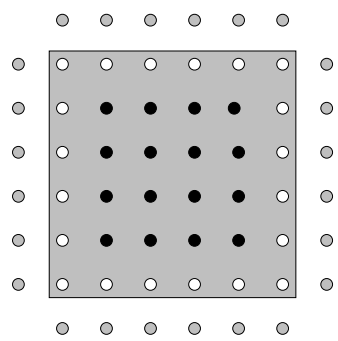

Fig. 1. Slice of a $6^{4}$ block $\Lambda$ (points inside the grey square) and its exterior boundary $\partial \Lambda$ (points outside the square) along a two-dimensional lattice plane. The white points in the square represent the interior boundary $\partial \Lambda^{*}$ of the block.

operator may be written in the form

$$
D_{\mathrm{w}}=\frac{1}{2}\left\{\gamma_{\mu}\left(\nabla_{\mu}^{*}+\nabla_{\mu}\right)-\nabla_{\mu}^{*} \nabla_{\mu}\right\}
$$

A sum over $\mu$ is implied here and the Dirac matrices $\gamma_{\mu}$ are taken to be hermitian. In the following, the Schwarz procedure will be set up for the linear equation

$$
D \psi(x)=\eta(x), \quad D \equiv D_{\mathrm{w}}+m_{0},
$$

in which $\eta$ is an arbitrary source field and $m_{0}$ the bare quark mass.

\subsection{Block terminology}

Like any other domain decomposition method, the Schwarz procedure operates on a covering of the lattice by a collection of domains. In the present context it is natural to choose the domains to be rectangular blocks of lattice points. Any such block is completely characterized by the lengths of its edges and by its position. In practice the blocks are usually taken to be fairly small, particularly on parallel computers, where for reasons of efficiency they should be contained in the local lattices. $\dagger$

The exterior boundary $\partial \Lambda$ of a given block $\Lambda$ is defined to be the set of all lattice points that have distance 1 from $\Lambda$ (see fig. 1). Each exterior boundary point has a closest "partner" point in the block. The interior boundary $\partial \Lambda^{*}$ of $\Lambda$ consists of all these points, and the set of points that are not in the block is denoted by $\Lambda^{*}$.

$†$ Parallel programs in lattice QCD are usually based on a division of the lattice into sublattices that are associated to the processors of the computer or to different program threads. The term "local lattice" refers to these sublattices. 
In position space the lattice Dirac operator is a sparse matrix that assumes the block-diagonal form

$$
D=\left(\begin{array}{ll}
D_{\Lambda} & D_{\partial \Lambda} \\
D_{\partial \Lambda^{*}} & D_{\Lambda^{*}}
\end{array}\right)
$$

if the lattice points are ordered so that those in $\Lambda$ come first. The operator $D_{\Lambda}$, for example, acts on quark fields on $\Lambda$ in precisely the same way as $D$, except that all terms involving the exterior boundary points are set to zero (which is equivalent to imposing Dirichlet boundary conditions on $\partial \Lambda$ ).

It is often convenient to consider $D_{\Lambda}, D_{\Lambda^{*}}, D_{\partial \Lambda}$ and $D_{\partial \Lambda^{*}}$ to operate on quark fields on the whole lattice rather than on fields that are defined on $\Lambda$ or $\Lambda^{*}$ only. The embedding is done in the obvious way by padding with zeros so that eq. (2.5), for example, may equivalently be written in the form

$$
D=D_{\Lambda}+D_{\Lambda^{*}}+D_{\partial \Lambda}+D_{\partial \Lambda^{*}}
$$

This notation is perhaps slightly abusive but it will usually be clear from the context which interpretation applies.

\subsection{Definition of the Schwarz procedure}

In the form in which it was originally conceived [4], the Schwarz procedure is assumed to operate on overlapping domains. The convergence of the method actually tends to be better the larger the overlaps are [1]. On the other hand, more domains are then required to cover the lattice, which slows down the computations, particularly in four dimensions, where the average occupation number is rapidly growing with the overlap size. If the Schwarz procedure is only used as a preconditioner (as will be the case here), it can thus be advantageous to choose the domains to be non-overlapping.

The obvious choice is then to cover the lattice by a regular grid of non-overlapping rectangular blocks of equal size such as the one shown in fig. 2. Although this is not required for the Schwarz procedure, it will be assumed, for technical reasons, that the block sizes are even and that the grid can be chessboard-coloured.

The so-called multiplicative Schwarz procedure now visits the blocks in the chosen block grid sequentially and updates the current approximation $\psi$ to the solution of the Wilson-Dirac equation (2.4) there. At the start of the iteration one can take $\psi=0$, for example. When the algorithm arrives at block $\Lambda$, the updated field $\psi^{\prime}$ is obtained by solving the equations

$$
\left.D \psi^{\prime}(x)\right|_{x \in \Lambda}=\eta(x),\left.\quad \psi^{\prime}(x)\right|_{x \notin \Lambda}=\psi(x) .
$$




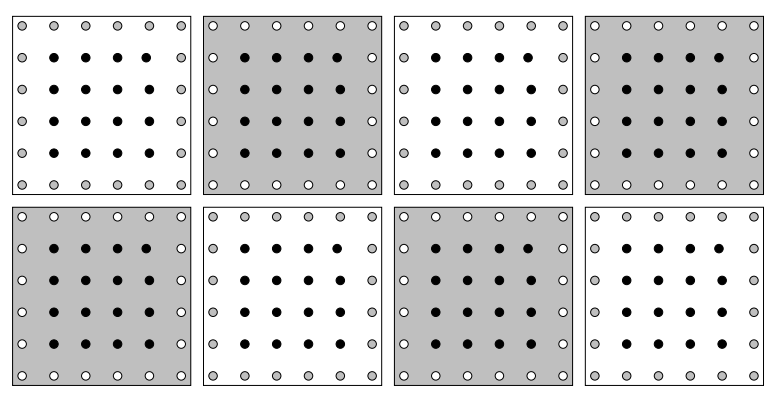

Fig. 2. Two-dimensional cross-section of a grid of non-overlapping $6^{4}$ blocks that covers a lattice of size $24 \times 12^{3}$. Such grids can be chessboard-coloured if there is an even number of blocks in all dimensions.

It is straightforward to check that this amounts to setting

$$
\psi^{\prime}=\psi+D_{\Lambda}^{-1}(\eta-D \psi)
$$

where it is understood that $D_{\Lambda}^{-1}$ acts on the restriction of the current residue $\eta-D \psi$ to the block $\Lambda$. This form of the iteration is numerically stable and is the one that is actually programmed.

\subsection{Schwarz iteration operator}

The structure of the Schwarz procedure becomes more transparent if it is rewritten in a slightly more compact form. To this end first note that the residue of the new approximation (2.8) coincides with the old residue, except on the block $\Lambda$ and on the neighbouring blocks. So if all black and all white blocks are visited alternatingly, it is clear that the order of the blocks with the same colour is irrelevant and that they can in fact be updated simultaneously.

Effectively the algorithm thus operates on only two domains, one being the union $\Omega$ of all black blocks and the other the union $\Omega^{*}$ of all white blocks. With respect to this division of the lattice, the Dirac operator decomposes into a sum of operators $D_{\Omega}, D_{\Omega^{*}}, D_{\partial \Omega}$ and $D_{\partial \Omega^{*}}$, as in the case of the division defined by a block $\Lambda$ and its complement $\Lambda^{*}$. Some algebra then shows that a complete Schwarz cycle, where the quark field is updated on all the black blocks followed by all the white blocks, amounts to the transformation

$$
\begin{aligned}
& \psi \rightarrow(1-K D) \psi+K \eta \\
& K \equiv D_{\Omega}^{-1}+D_{\Omega^{*}}^{-1}-D_{\Omega^{*}}^{-1} D_{\partial \Omega^{*}} D_{\Omega}^{-1} .
\end{aligned}
$$


The field thus evolves from $\psi=0$ to

$$
\psi=K \sum_{\nu=0}^{n_{\mathrm{cy}}-1}(1-D K)^{\nu} \eta
$$

after $n_{\text {cy }}$ cycles, which shows that the Schwarz procedure attempts to obtain the solution in the form of a Neumann series with iteration operator $1-D K$. From this point of view, the operator $K$ plays the rôle of a preconditioner for $D$.

\section{Preconditioned GCR algorithm}

Some numerical experimenting suggests that the spectral radius of the iteration operator $1-D K$ is bounded by 1 , at least as long as $D$ is not exceptionally ill-conditioned. The rate of convergence of the Schwarz procedure is often disappointing, however, and it is thus advisable to combine the method with a Krylov space accelerator such as the generalized conjugate residual (GCR) algorithm.

\subsection{Schwarz preconditioner}

The basic idea is to use the Schwarz procedure as a preconditioner rather than as a solver, and to apply the GCR algorithm to the preconditioned system. As discussed in the previous section, the Schwarz procedure can be seen as an operation acting on the source field, which produces an approximate solution of eq. (2.4), for any specified number $n_{\text {cy }}$ of cycles. If the associated linear operator is denoted by $M_{\text {sap }}$, the right-preconditioned equation

$$
D M_{\mathrm{sap}} \phi=\eta
$$

is thus expected to be better conditioned than the original linear system (once this equation is solved, the solution of the latter is obtained by setting $\psi=M_{\text {sap }} \phi$ ).

From eq. (2.11) it follows that $M_{\text {sap }}$ is given explicitly by

$$
M_{\mathrm{sap}}=K \sum_{\nu=0}^{n_{\mathrm{cy}}-1}(1-D K)^{\nu} .
$$

In practice this formula is not used, however, and the calculation of (say) $\psi=M_{\text {sap }} \phi$ instead proceeds along the lines described in subsect. 2.3 , i.e. by starting from $\psi=0$ 
and running through the blocks in the block grid, updating $\psi$ on each block, with $\phi$ now being the source.

\subsection{Outline of the GCR algorithm}

The GCR algorithm is a Krylov space solver that is mathematically equivalent to the GMRES algorithm (see ref. [2] for example). It offers some technical advantages with respect to the latter if the preconditioner is implemented approximately, as will be the case here. In the following a variant of the GCR algorithm is described that was introduced in the context of the so-called GMRESR algorithm $[8,9]$.

Starting from $\psi_{0}=0$, the GCR algorithm generates a sequence of approximate solutions $\psi_{k}, k \geq 0$, of the Wilson-Dirac equation (2.4) recursively. The associated residues are defined by

$$
\rho_{k}=\eta-D \psi_{k}
$$

and the recursion then chooses $\psi_{k+1}$ to be the field in the linear subspace spanned by $\psi_{1}, \ldots, \psi_{k}, M_{\text {sap }} \rho_{k}$, which minimizes $\left\|\rho_{k+1}\right\|$.

It is important to understand that the field $M_{\text {sap }} \rho_{k}$ merely defines the direction in which the previous subspace is extended. In particular, a precise implementation of the preconditioner is not really required, and its definition may even vary from step to step. The worst that can happen in this case is that the convergence of the algorithm slows down, but the algorithm will never become unstable or otherwise invalid.

\subsection{Definition of the GCR recursion}

The GCR algorithm generates two sequences of fields, $\chi_{k}$ and $\xi_{k}$, that must be kept in the memory of the computer together with the last residue $\rho_{k}$. A special feature of the chosen implementation is that the approximate solution $\psi_{k}$ itself does not take part in the recursion and is only constructed at the end of the calculation. For reasons of efficiency the recursion may have to be restarted after a certain number of steps, taking the last residue as the new source. This point will be discussed again in sect. 4, together with other implementation details.

Apart from the sequences of fields, the recursion generates sequences of complex numbers $a_{l k}, b_{k}$ and $c_{k}$ that should also be preserved. An explicit description of the algorithm is provided by the pseudo-code displayed in fig. 3. Note that the fields $\chi_{k}$ satisfy

$$
\left(\chi_{i}, \chi_{j}\right)=\delta_{i j}, \quad 0 \leq i, j \leq k,
$$




$$
\begin{aligned}
& \rho_{0}=\eta \\
& \text { for } k=0,1,2, \ldots \text { do } \\
& \quad \xi_{k}=M_{\text {sap }} \rho_{k} \\
& \quad \chi_{k}=D \xi_{k} \\
& \text { for } l=0, \ldots, k-1 \text { do } \\
& \quad a_{l k}=\left(\chi_{l}, \chi_{k}\right) \\
& \quad \chi_{k}=\chi_{k}-a_{l k} \chi_{l} \\
& \text { end do } \\
& b_{k}=\left\|\chi_{k}\right\| \\
& \chi_{k}=\chi_{k} / b_{k} \\
& c_{k}=\left(\chi_{k}, \rho_{k}\right) \\
& \rho_{k+1}=\rho_{k}-c_{k} \chi_{k} \\
& \text { end do }
\end{aligned}
$$

Fig. 3. Pseudo-code for the GCR recursion. On the first line in the outer loop the Schwarz preconditioner is applied to the current residue $\rho_{k}$. For the calculation of the next residue in the last two lines, the auxiliary field $\chi_{k}$ first needs to be constructed through a Gram-Schmidt orthogonalization process (inner loop).

by the time $\rho_{k+1}$ is calculated. The latter is then the projection of the first residue $\eta$ to the orthogonal complement of the space spanned by the fields $\chi_{0}, \ldots, \chi_{k}$, which coincides with the space spanned by the fields $D \xi_{0}, \ldots, D \xi_{k}$. In other words,

$$
\rho_{k+1}=\eta-\sum_{l=0}^{k} \alpha_{l} D \xi_{l}
$$

for some complex coefficients $\alpha_{k}$ chosen such that $\left\|\rho_{k+1}\right\|$ is minimized. This shows that $\rho_{k+1}$ is indeed the residue of the approximate solution $\psi_{k+1}$ that was defined in subsect. 3.2, and the pseudo-code thus implements the GCR algorithm correctly.

\subsection{Calculation of $\psi_{k+1}$}

When it is decided to stop or restart the GCR recursion after $k+1$ steps, the field $\psi_{k+1}$ will have to be computed. From the above it is obvious that

$$
\psi_{k+1}=\sum_{l=0}^{k} \alpha_{l} \xi_{l}
$$


but the coefficients $\alpha_{l}$ [which are the same as in eq. (3.5)] are only implicitly known at this point. They can, however, be calculated by noting that the fields $\chi_{l}$ satisfy

$$
D \xi_{l}=b_{l} \chi_{l}+\sum_{i=0}^{l-1} a_{i l} \chi_{i}, \quad l=0,1, \ldots, k
$$

and also

$$
\rho_{k+1}=\eta-\sum_{l=0}^{k} c_{l} \chi_{l} .
$$

Now if $\rho_{k+1}=\eta-D \psi_{k+1}$ is evaluated by first inserting eq. (3.6) and then eq. (3.7), the comparison with eq. (3.8) leads to the linear system

$$
b_{l} \alpha_{l}+\sum_{i=l+1}^{k} a_{l i} \alpha_{i}=c_{l}, \quad l=k, k-1, \ldots, 0,
$$

which can be solved straightforwardly for the coefficients $\alpha_{l}$ by back-substitution.

\section{Numerical implementation of the Schwarz procedure}

In this section some further details of the proposed algorithm are discussed, leaving away any purely programming issues. The Schwarz procedure is actually not entirely trivial to program, and a good choice of the data and program structures can be important (see appendix A for some suggestions).

\subsection{Block solver}

The first question to be addressed here is how to perform the inversion of the local Wilson-Dirac operator $D_{\Lambda}$ in the update step (2.8). Clearly an exact inversion is not possible in practice, and is in fact not needed, since an inaccurate implementation of the Schwarz preconditioner $M_{\text {sap }}$ is perfectly acceptable (cf. subsect. 3.2).

Perhaps the simplest iterative solver that may be used in this context is the minimal residual algorithm (ref. [2], §5.3.2). Independently of the value of the quark mass, only few iterations then are in general sufficient to reach the point where the rate of convergence of the GCR solver is not significantly improved by performing 
further iterations. This is so in part because $D_{\Lambda}$ tends to be well-conditioned, but it should also be noted that the Schwarz preconditioner is applied when the accuracy of the current approximate solution $\psi_{k}$ is anyway limited.

The update step (2.8) takes the current residue $\rho=\eta-D \psi$ as input and modifies the field $\psi$ on the block $\Lambda$. It is thus an operation that acts on these two globally defined fields in the following way:

(a) The restriction of the residue $\rho$ to the block $\Lambda$ is copied to a block field $\rho_{\Lambda}$. An approximate solution $\zeta_{\Lambda}$ of the block equation $D_{\Lambda} \zeta_{\Lambda}=\rho_{\Lambda}$ is then obtained by applying a fixed number $n_{\mathrm{mr}}$ of minimal residual iterations.

(b) $\psi$ is replaced by $\psi+\zeta_{\Lambda}$ on $\Lambda$ and is left untouched elsewhere. The residue $\rho$ is also updated by adding $\tilde{\rho}_{\Lambda}=\rho_{\Lambda}-D_{\Lambda} \zeta_{\Lambda}$ on $\Lambda$ and subtracting $D_{\partial \Lambda^{*}} \zeta_{\Lambda}$ on $\partial \Lambda$. Note that the minimal residual algorithm returns both the approximate solution $\zeta_{\Lambda}$ and the associated residue $\tilde{\rho}_{\Lambda}$.

Besides $M_{\text {sap }} \eta$ this implementation of the Schwarz procedure yields $D M_{\text {sap }} \eta$ essentially for free. There are two parameters, the number $n_{\text {cy }}$ of Schwarz cycles and the number $n_{\mathrm{mr}}$ of minimal residual iterations performed in step (a), which should be adjusted so as to minimize the average computer time needed to obtain the solution of eq. (2.4) to the desired accuracy.

\subsection{Even-odd preconditioning}

If the Schwarz blocks are chosen to have an even number of points in all dimensions, even-odd preconditioning may be applied to accelerate the block solver. This simple modification is certainly worth the additional programming effort, since most of the computer time is spent in step (a).

On the full lattice, the even-odd preconditioned form of the Wilson-Dirac equation (2.4) reads

$$
\begin{aligned}
& \hat{D} \psi_{\mathrm{e}}=\eta_{\mathrm{e}}-D_{\mathrm{eo}}\left(D_{\mathrm{oo}}\right)^{-1} \eta_{\mathrm{o}}, \\
& \hat{D} \equiv D_{\mathrm{ee}}-D_{\mathrm{eo}}\left(D_{\mathrm{oo}}\right)^{-1} D_{\mathrm{oe}}
\end{aligned}
$$

where the subscripts "e" and "o" refer to the even and the odd lattice sites. Equation (4.1) determines $\psi_{\mathrm{e}}$, while the solution on the odd points,

$$
\psi_{\mathrm{o}}=\left(D_{\mathrm{oo}}\right)^{-1}\left(\eta_{\mathrm{o}}-D_{\mathrm{oe}} \psi_{\mathrm{e}}\right)
$$

is obtained algebraically. 
All these formulae carry over to the block equation $D_{\Lambda} \zeta_{\Lambda}=\rho_{\Lambda}$ without any modifications. In particular, the even-odd preconditioned Wilson-Dirac operator $\hat{D}_{\Lambda}$ is again given by eq. (4.2), with $D$ replaced by $D_{\Lambda}$. The minimal residual algorithm may then be used to solve the preconditioned system, in which case steps (a) and (b) together require the equivalent of $n_{\mathrm{mr}}+1$ applications of $\hat{D}_{\Lambda}$ (if $n_{\mathrm{mr}}$ iterations are performed) plus the calculation of about $2 n_{\mathrm{mr}}$ scalar products and linear combinations.

\subsection{Communication overhead}

On parallel computers each processor operates on a sublattice that is usually taken to be a rectangular block similar to the blocks on which the Schwarz procedure operates. In order to minimize the communication overhead, the local lattice should obviously be divisible by the Schwarz blocks. The block solver does not require any communications in this case, and it is only in step (b), when $D_{\partial \Lambda^{*}} \zeta_{\Lambda}$ is subtracted from the current residue, that some data need to be exchanged. In terms of "communicated words per arithmetic operation", a reduction by a factor $n_{\mathrm{mr}}+1$ is thus achieved relative to the overhead in the program for the Wilson-Dirac operator.

Another factor of 2 in the communication overhead can actually be saved by noting that

$$
\left(1-n_{\mu} \gamma_{\mu}\right) D_{\partial \Lambda^{*}} \zeta_{\Lambda}(x)=0 \quad \text { for all } \quad x \in \partial \Lambda
$$

where $n_{\mu}$ is the outward normal vector to the boundary $\partial \Lambda$ at $x$. There are thus two linear relations among the four Dirac components of $D_{\partial \Lambda^{*}} \zeta_{\Lambda}(x)$, and it is therefore sufficient to communicate the upper two components in the cases where a communication is required. $\dagger$

Taking this into account, the update of the residue in step (b) proceeds by first adding $\tilde{\rho}_{\Lambda}$ on all points in the block. The upper two components of $D_{\partial \Lambda^{*}} \zeta_{\Lambda}$ are then calculated and stored in an auxiliary array. Some parts of this field may reside on the exterior boundary of the local lattice, in which case they have to be communicated to the appropriate neighbouring processes. Thereafter the full Dirac spinors $D_{\partial \Lambda^{*}} \zeta_{\Lambda}$ are reconstructed, using eq. (4.4), and subtracted from the residue. Note that the communication and subtraction can be delayed until all black (or all white) blocks have been visited. This allows the relevant parts of the fields on the boundaries of all the black (white) blocks to be transferred in a single data package per direction.

$\dagger$ It is assumed here that the Dirac matrices are in a chiral representation, where $\gamma_{5}=\gamma_{0} \gamma_{1} \gamma_{2} \gamma_{3}$

is a diagonal matrix with entries $1,1,-1,-1$ on the diagonal. The upper two components of the boundary field $D_{\partial \Lambda^{*}} \zeta_{\Lambda}$ are then guaranteed to be linearly independent. 


\subsection{Non-algorithmic accelerations}

Since the Schwarz preconditioner does not need to be accurately implemented, one may just as well use single-precision arithmetic in the program for the block solver. The GCR algorithm may also be coded in this way, with scalar products accumulated in double precision, provided it is restarted before the residue has decreased by more than a few orders of magnitude. Evidently, at each restart of the algorithm, the last residue should be recalculated using double-precision arithmetic to ensure that the solution of the Wilson-Dirac equation is obtained to full precision (see ref. [10] for a more detailed discussion of this point).

All current PC processors support vector instruction sets (SSE, SSE2, 3DNow! or AltiVec) that allow short vectors of floating-point numbers to be processed simultaneously [11]. The block solver and other time-critical parts of the program can be significantly accelerated using these additional capabilities, especially so if the fields on the Schwarz blocks fit into the cache of the processors.

\section{Tests of the algorithm}

As is generally the case for domain decomposition methods, the proposed algorithm is expected to perform particularly well on parallel computers. At this point nothing is known, however, about the convergence properties of the algorithm in the relevant range of parameters. The tests reported in this section serve to fill this gap and to show that an excellent parallel efficiency is indeed achieved on the computer where the tests were carried out (see appendix B for the technical specifications).

\subsection{Choice of parameters}

Different parameter sets have been considered, but most results were obtained for a particular choice, defined in the following lines, that will be referred to as the default parameter set. In general the strategy of the tests is to distribute the computations required for the solution of the Wilson-Dirac equation on a fixed lattice over an increasing number of processors, so that the effects of the communication overhead can be clearly seen. Taking this into account, and the fact that the available computer has 64 processors, it was decided to perform the tests on a $16^{4}$ lattice.

Samples of 100 statistically independent gauge field configurations were then generated, using the Wilson plaquette action at (inverse) coupling $\beta=5.9$, where the lattice spacing is about $0.10 \mathrm{fm}$. In each test run, five values of the bare quark mass 
Table 1. Timings in $\mu s$ [speed in Gflop/s] per lattice point and application of $\hat{D}$

\begin{tabular}{clcccc}
\hline $\begin{array}{c}\text { number of } \\
\text { processors }\end{array}$ & $\begin{array}{c}\text { local } \\
\text { lattice }\end{array}$ & $\left(M_{\text {sap }} \psi\right)_{32 \text { bit }}$ & $(\hat{D} \psi)_{64 \text { bit }}$ & SAP+GCR & BiCGstab \\
\hline 1 & $16^{4}$ & $0.64[2.35]$ & $1.39[0.98]$ & 0.75 & 2.2 \\
2 & $16^{3} \times 8$ & $0.86[1.74]$ & $2.59[0.52]$ & 1.12 & 4.2 \\
4 & $16^{2} \times 8^{2}$ & $0.86[1.74]$ & $3.00[0.45]$ & 1.10 & 4.7 \\
8 & $16 \times 8^{3}$ & $0.88[1.71]$ & $3.42[0.40]$ & 1.11 & 5.2 \\
16 & $8^{4}$ & $0.90[1.65]$ & $4.17[0.33]$ & 1.13 & 5.8 \\
32 & $8^{3} \times 4$ & $0.94[1.58]$ & $4.76[0.28]$ & 1.24 & 6.7 \\
64 & $8^{2} \times 4^{2}$ & $0.98[1.53]$ & $5.50[0.25]$ & 1.21 & 7.3 \\
\hline
\end{tabular}

$m_{0}$ were considered, corresponding to values of $\kappa=\left(8+2 m_{0}\right)^{-1}$ equal to 0.1566 , $0.1574,0.1583,0.1589$ and 0.1592 . According to a recent large-scale study by the CP-PACS collaboration [12], the "pion" mass $m_{\pi}$ decreases from about $579 \mathrm{MeV}$ to $320 \mathrm{MeV}$ in this range of quark masses.

The sizes of the Schwarz blocks are constrained by the requirement that they should divide the local lattices. Larger blocks tend to yield a better preconditioner, but the block solver then consumes more processor time per lattice point. The exact choice is not critical, and a block size of $8 \times 4^{3}$ appears to be a good compromise for the given lattice and machine parameters. As for the remaining free parameters of the algorithm, some experimenting suggests to set $n_{\mathrm{mr}}=4, n_{\mathrm{cy}}=5$ and $n_{\mathrm{kv}}=16$, where the latter limits the number of Krylov vectors that are generated by the GCR recursion before it is restarted.

\subsection{Reference algorithm}

To be able to judge the overall performance of the new algorithm, timings were also taken for the BiCGstab algorithm [13], which is probably the fastest Krylov space solver for the Wilson-Dirac equation [14]. The algorithm is applied to the even-odd preconditioned system (4.1), using a highly optimized code. In particular, as in the case of the Schwarz procedure discussed in subsect. 4.3, the program only transfers the upper two components of the Dirac spinors that need to be communicated, thus saving a factor of 2 in the communication overhead.

A technical detail worth mentioning here is that single-precision acceleration [10] does not work too well in this case, because the increased arithmetic speed is practi- 


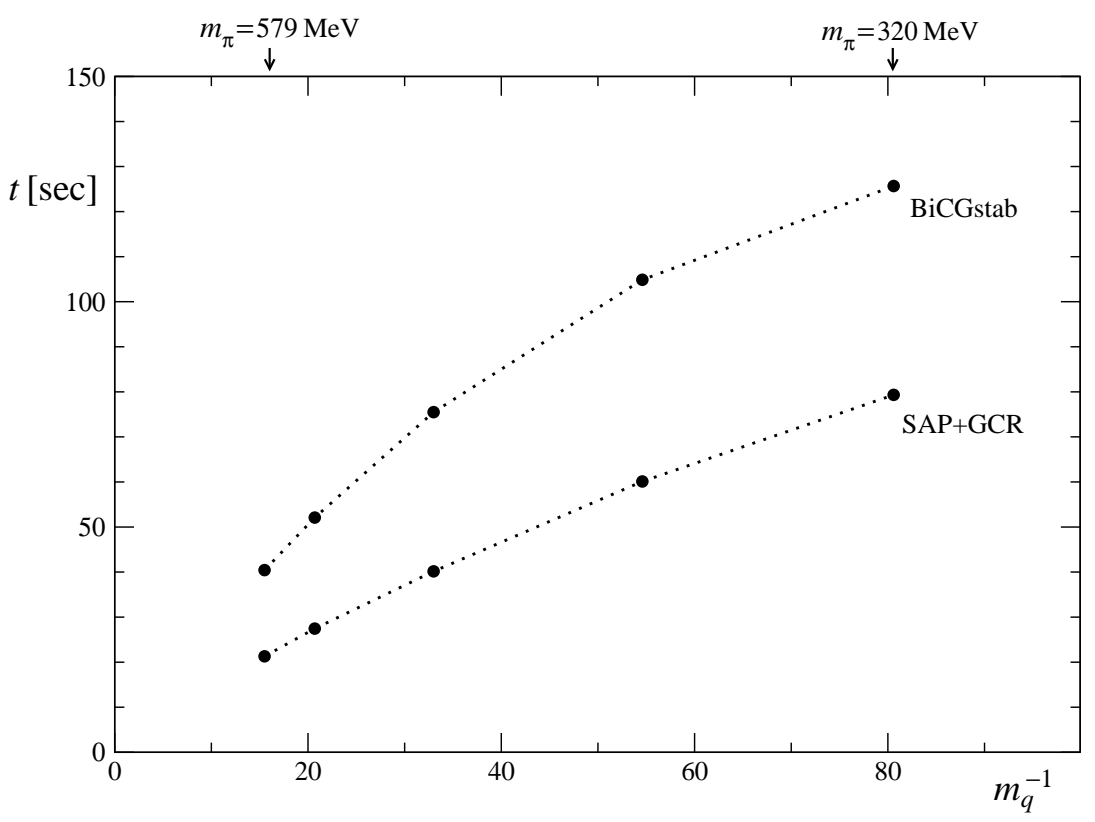

Fig. 4. Average execution time $t$ needed to solve the Wilson-Dirac equation (2.4) as a function of the bare quark mass. A single processor is used here and the solvers are stopped when $\|\eta-D \psi\|<10^{-8}\|\eta\|$. The lattice and algorithmic parameters are as specified in subsect. 5.1, and the dotted lines are drawn to guide the eye.

cally compensated by the fact that more iterations are required to reach a specified precision. Moreover, there is a negative effect on the stability of the algorithm. The BiCGstab solver was therefore implemented in double-precision arithmetic, with all time-critical parts programmed using SSE2 vector instructions.

\subsection{Basic program and algorithm performance}

In terms of numbers of floating-point operations, the Schwarz preconditioner $M_{\text {sap }}$ is roughly equivalent to $\left(n_{\mathrm{mr}}+1\right) n_{\mathrm{cy}}$ applications of $\hat{D}$. The speed of the programs that implement the preconditioner and the complete solver (denoted "SAP+GCR" in the following) is therefore best measured in units of execution time per local lattice point and equivalent application of $\hat{D}$.

For the case where a single processor is used, some relevant performance figures are quoted in the first line of table 1. The associated Gflop/s rates, given in square brackets, refer to the actual number of floating-point operations. It should again be emphasized at this point that the use of single-precision arithmetic for the Schwarz preconditioner is entirely adequate and does not set a limit on the precision that can 


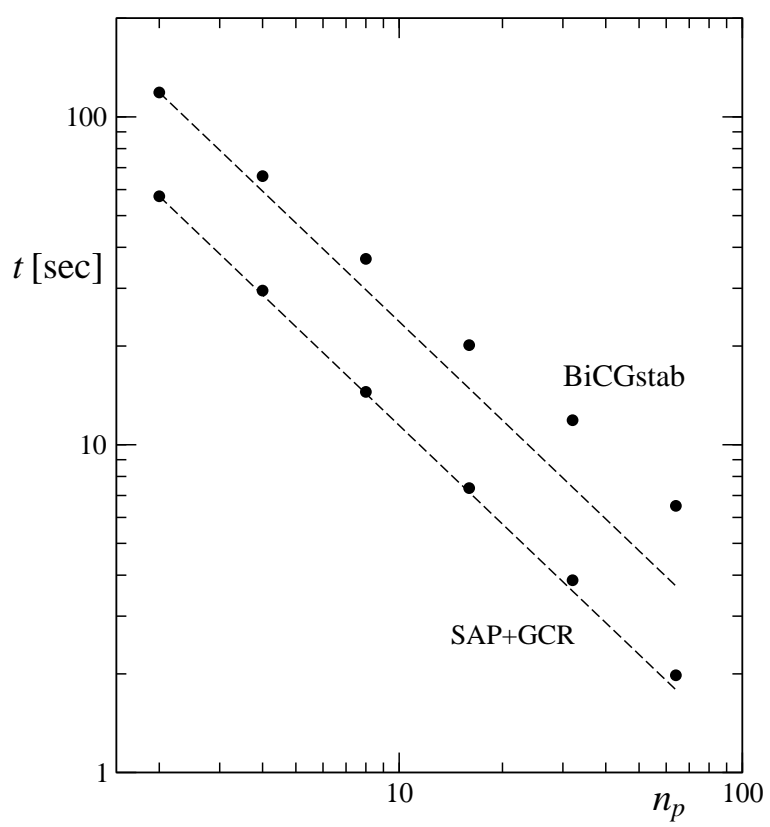

Fig. 5. Execution time required for the solution of the Wilson-Dirac equation (2.4) as a function of the number $n_{p}=2,4, \ldots, 64$ of processors used by the program. The parameters are as in table 1 and fig. 4 , with the quark mass fixed to the smallest value considered there. Ideal parallel scaling is represented by the dashed lines.

be reached by the SAP+GCR solver. Note that a BiCGstab iteration involves two applications of $\hat{D}$, so that the time per iteration and lattice point is twice the figure quoted in table 1. The linear algebra in the BiCGstab algorithm thus consumes more than a third of the total time spent by the single-processor program. This overhead is much smaller in the case of the $\mathrm{SAP}+\mathrm{GCR}$ solver, because the application of the Schwarz preconditioner is relatively expensive in terms of computer time.

In fig. 4 the time needed for the solution of the Wilson-Dirac equation to a specified precision is plotted versus $1 / m_{q}$, where $m_{q}=m_{0}-m_{c}$ denotes the additively renormalized quark mass and $m_{c}$ the critical bare mass. The SAP+GCR solver thus obtains the solution faster than the BiCGstab algorithm, by a factor ranging from 1.6 to 1.9, if a single processor is used. When going from large to small quark masses, the curvature in the data suggests that the BiCGstab solver becomes relatively more efficient, but this trend is less pronounced on larger lattices and should therefore be taken as a finite-volume anomaly (cf. subsect. 5.5).

Another result of these tests is that the GCR algorithm converges in comparatively few steps. On average only 17 Krylov vectors are generated at the largest quark mass 


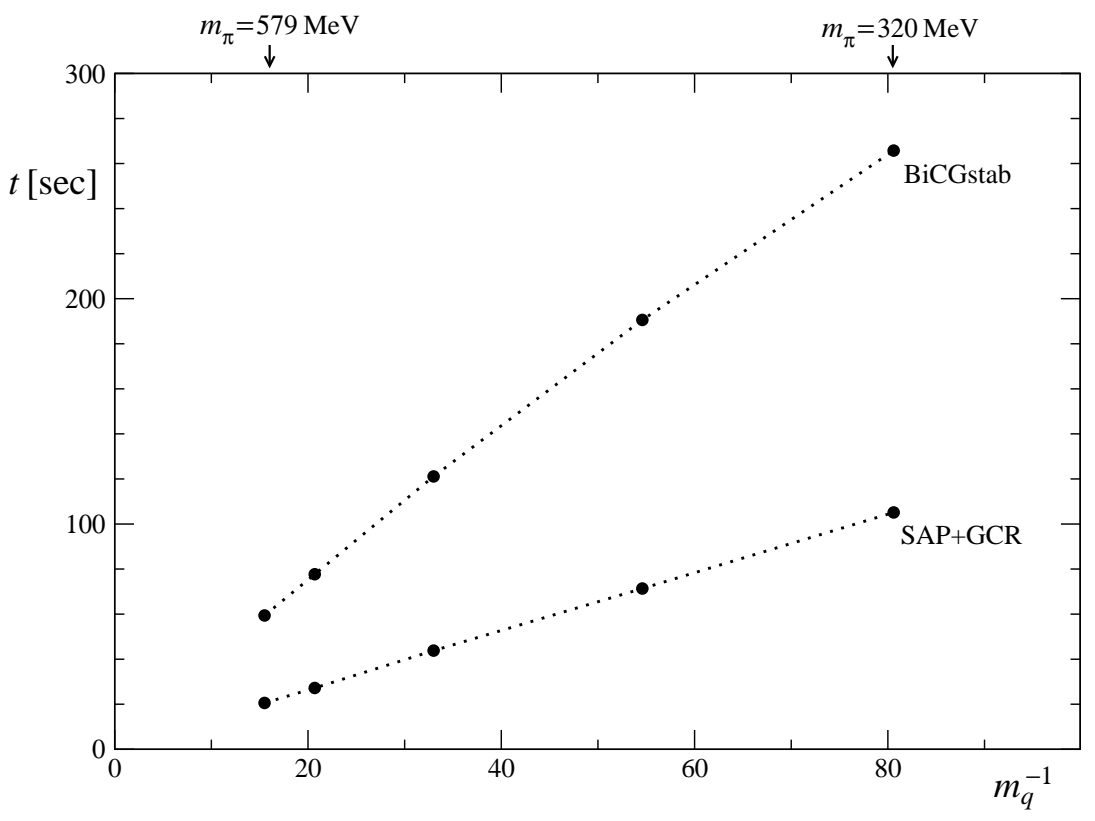

Fig. 6. Same as fig. 4, but now for a $48 \times 24^{3}$ lattice distributed over 16 processors. The sizes of the local lattices and the Schwarz blocks are $24 \times 12^{3}$ and $6^{2} \times 4^{2}$ respectively, and $n_{\mathrm{kv}}$ has been set to 24 in this case. All other parameters are unchanged.

and 64 at the smallest mass, while 140 to 437 BiCGstab iterations are needed to obtain the solution to the specified precision. Since both algorithms have similar theoretical convergence properties [2], this suggests that the condition number of the Schwarz preconditioned Wilson-Dirac operator $D M_{\text {sap }}$ is roughly 15 times smaller than the condition number of $\hat{D}$, at all quark masses that have been considered.

\subsection{Parallel efficiency}

Evidently these algorithmic properties are unchanged when the computational work is distributed over several processors, and the variations in the performance figures listed in table 1 thus provide a direct measure for the parallelization losses. The lattice and algorithmic parameters have been set to the default values in all these tests. In particular, the dimensionality of the parallelization and the communication overhead are increasing as one moves down the table.

When going from 1 to 2 processors, the steep rise in the timings is, however, only partially due to the communication overhead. The principal cause for the slow-down instead is the fact that the two processors on the nodes of the computer share the available memory bandwidth (cf. appendix B). For this reason the parallel efficiency 
of the programs should be judged by comparing the many-processor timings with the performance of the two-processor programs rather than the single-processor ones.

The results quoted in table 1 show that the parallel efficiency of the programs for the Schwarz preconditioner and the SAP+GCR algorithm is very good. If the lattice is distributed over all 64 processors, for example, the surface-to-volume ratio of the local lattices is as large as 1.5, but the parallelization overhead in the program for the preconditioner nevertheless only consumes about $12 \%$ of the execution time. Moreover, this figure is even smaller in the case of the solver, because practically no communications are required in the linear algebra programs. Note incidentally that the computer achieves a total sustained speed of nearly $100 \mathrm{Gflop} / \mathrm{s}$ at this point.

Given the available network bandwidth, it is no surprise, however, that the program for the even-odd preconditioned lattice Dirac operator and the BiCGstab solver do not scale as well. The difference is perhaps made clearer by fig. 5 , where the time needed to solve the Wilson-Dirac equation is plotted as a function of the number of processors. In particular, if all 64 processors are used, and depending on the quark mass, the SAP+GCR solver is from 3.3 to 4.1 times faster than the BiCGstab solver.

\subsection{Larger lattices}

The timings taken on larger lattices suggest that the performance pattern remains practically the same as the one seen so far. Essentially the communication losses are determined by the surface-to-volume ratio of the local lattices, while the number of processors that are used appears to be less relevant.

For illustration, the time needed to solve the Wilson-Dirac equation on a $48 \times 24^{3}$ lattice is plotted in fig. 6 . In this test 16 processors were used and the surface-tovolume ratio of the local lattices was only 0.6. The plot shows that the SAP+GCR and the BiCGstab algorithms have a similar scaling behaviour as a function of the quark mass, the SAP+GCR solver being faster by a factor ranging from 2.5 to 2.9 . Moreover, the ratio of the iteration counts of the two algorithms is not very different from the one observed before, although the convergence is generally slower on this lattice than on the $16^{4}$ lattice.

\section{Conclusions}

The algorithm presented in this paper provides the first example for a successful ap-

plication of a domain decomposition method in lattice QCD. Traditionally the lattice 
Dirac equation is solved using a general purpose algorithm such as the BiCGstab solver. Domain decomposition methods are fundamentally different from these, because they do not operate in the usual Krylov spaces. The locality and ellipticity of the equation are instead exploited to construct a highly effective preconditioner.

An obvious advantage of domain decomposition methods is that they are usually well suited for parallel processing. In the case of the algorithm described here, for example, the number of words per arithmetic operation which must be communicated is smaller than the communication rate in the BiCGstab algorithm by about a factor 5. This may not be an impressive figure, but it should be noted in this connection that a reduction of the communication overhead by a factor 2 alone allows 16 times more processors to be used efficiently once the program is parallelized in four dimensions (and runs well in this mode).

In terms of condition numbers, the non-overlapping Schwarz procedure that was considered in this paper yields an excellent preconditioner for the Wilson-Dirac operator. The numerical tests showed this very clearly, but another outcome of the tests was that the scaling behaviour of the SAP+GCR solver with respect to the quark mass is rather similar to the one of the BiCGstab algorithm. It is conceivable that more sophisticated domain decomposition methods can do better than this, and there are in fact quite a few cases in other branches of science, where such methods come close to having an ideal (i.e. constant) scaling behaviour [1]. For lattice QCD any progress in this direction would evidently be a major step forward.

The numerical work reported in this paper was carried out on a PC-cluster at the Institut für Theoretische Physik der Universität Bern, which was funded in part by the Schweizerischer Nationalfonds. I wish to thank both institutions for the support given to this project and Peter Weisz for a critical reading of the manuscript.

\section{Appendix A. Programming issues}

The remarks collected in this appendix provide some guidelines for the programming of the Schwarz procedure. They are based on the experience gained in the course of this work, where all programs were written in C, using the standard message passing interface (MPI) for the communications between the processors. Evidently, the recommended strategies may not be appropriate in other programming environments. 


\section{A.1 Object orientation}

The Schwarz procedure is an obvious case for an object-oriented approach. A block of lattice points, for example, is best represented by a structure that contains the block fields and the local geometry arrays. There should be enough predefined information in this structure that the block solver can proceed without reference to the surrounding lattice. In particular, it is advantageous to have a copy of the relevant gauge field variables on each block. Other useful objects are block boundaries and the block grids on which the Schwarz procedure operates.

Among the members of the block and boundary structures, the geometry arrays play a central rôle. Lattice points are usually labelled by an integer index ix in an arbitrary way. The embedding in the global lattice of a block point with local index ix thus amounts to specifying its global index imb[ix]. Similarly each boundary point with local index ix has a unique partner point in the block with block index ipp [ix]. This approach views blocks, boundaries and the global lattice as nothing more than ordered sets of points, whose geometry is encoded in the appropriate index arrays.

Once the structures representing the objects have been defined, some utility programs need to be written for object creation, destruction and other basic manipulations. In particular, since blocks have their own fields, there must be functions that copy the relevant parts of the global fields to and from a specified block.

\section{A.2 Generic programs}

The programming effort as well as the program sizes can be significantly reduced by writing generic programs. Linear algebra routines, for example, only need to know the starting addresses of the spinor fields on which they operate and the number of spinors in the fields. The same programs can then be applied independently of the geometric context.

In the case of the programs for the Wilson-Dirac operator, a generic code requires as input the addresses of the gauge field, of the source and the target spinor fields, and of the nearest-neighbour index array. Evidently the quark mass and the number of elements in the fields must also be specified, but apart from this the program does not need any further information to be able to proceed. Different local geometries and boundary conditions are then easily taken into account through predefined index arrays.

\section{A.3 Parallelization}

If the global lattice is to be distributed over a large number of processors, it will be 
preferable, in general, to parallelize the program in four dimensions. Communications in eight directions are required in this case and some care should obviously be taken to ensure that the associated overhead remains small.

Data transfers via MPI send and receive functions are characterized by the pointto-point bandwidth of the network and the start-up latency. The latter can be kept to a minimum by using so-called persistent communication requests and by aligning the data arrays in memory so that any unnecessary data packing is avoided. It may actually be quite important to follow this advice, since the data packages that are exchanged in the course of the Schwarz procedure tend to be relatively small. In particular, advantage should be taken of the chessboard colouring of the Schwarz blocks to maximize the package sizes, as explained at the end of subsect. 4.3.

\section{Appendix B. Machine parameters}

The tests reported in sect. 5 were performed on a commodity PC-cluster with 32 nodes, each consisting of a motherboard with Intel E7500 chipset, two Intel Xeon processors (2.4 GHz, $512 \mathrm{~KB}$ cache), $1 \mathrm{~GB}$ of shared ECC DDR-200 memory and a Myrinet-2000 communication card. Fibre cables connect the Myrinet cards to a fast switch, which allows for arbitrary bidirectional point-to-point communications between the nodes.

The tested programs are written in $\mathrm{C}$ with all communications (including intranode data transfers) programmed using standard MPI functions. In most cases the best performance is obtained by having one process on each processor. In particular, if the program is written so that the two processors on the nodes send and receive data in an alternating fashion, the bidirectional bandwidth of the Myrinet network can then be saturated without any further programming effort.

On the software side, the gcc compiler version 3.2.2 was used with optimization options -mcpu=i586 -malign-double -fno-force-mem -0. Following ref. [11], SSE and SSE2 vector instructions were included through embedded assembler statements. The programs were then linked to the MPICH-over-GM library provided by Myricom to enable the MPI functionality.

In linear algebra programs (where the time required for the arithmetic operations is negligible), the effective total memory-to-processor bandwidth on each node is in the range from 1.6 to $2.4 \mathrm{~GB} / \mathrm{s}$. For bidirectional node-to-node communications and data packages larger than $1 \mathrm{MB}$, the network achieves a throughput of $160 \mathrm{MB} / \mathrm{s}$ in 
each direction. If persistent communication requests are used (in MPI parlance), the effective bandwidth per link and direction is still $92 \mathrm{MB} / \mathrm{s}$ for packages as small as $4 \mathrm{~KB}$. Unidirectional data transfers are generally faster and reach about $240 \mathrm{MB} / \mathrm{s}$.

\section{References}

[1] A. Quarteroni, A. Valli, Domain decomposition methods for partial differential equations (Oxford University Press, Oxford, 1999)

[2] Y. Saad, Iterative methods for sparse linear systems, 2nd ed. (SIAM, Philadelphia, 2003); see also http://www-users.cs.umn.edu/ ${ }^{\sim}$ saad/

[3] M. Lüscher, JHEP 0305 (2003) 052

[4] H. A. Schwarz, Gesammelte Mathematische Abhandlungen, vol. 2 (Springer Verlag, Berlin, 1890)

[5] K. G. Wilson, Phys. Rev. D10 (1974) 2445

[6] B. Sheikholeslami, R. Wohlert, Nucl. Phys. B 259 (1985) 572

[7] M. Lüscher, S. Sint, R. Sommer, P. Weisz, Nucl. Phys. B478 (1996) 365

[8] H. A. van der Vorst, C. Vuik, Num. Lin. Alg. Appl. 1 (1994) 369

[9] C. Vuik, J. Comput. Appl. Math. 61 (1995) 189

[10] L. Giusti, C. Hoelbling, M. Lüscher, H. Wittig, Comput. Phys. Commun. 153 (2003) 31

[11] M. Lüscher, Nucl. Phys. B (Proc. Suppl.) 106 (2002) 21

[12] S. Aoki et al. (CP-PACS collab.), Phys. Rev. D67 (2003) 034503

[13] H. A. van der Vorst, SIAM J. Sci. Stat. Comput. 13 (1992) 631

[14] A. Frommer, V. Hannemann, B. Nöckel, T. Lippert, K. Schilling, Int. J. Mod. Phys. C5 (1994) 1073 